\title{
Cantilever Sensors for Rapid Optical Antimicrobial Sensitivity Testing
}

\author{
Isabel Bennett, Alice L. B. Pyne,* and Rachel A. McKendry*
}

Cite This: ACS Sens. 2020, 5, 3133-3139

Read Online

ABSTRACT: Growing antimicrobial resistance (AMR) is a serious global threat to human health. Current methods to detect resistance include phenotypic antibiotic sensitivity testing (AST), which measures bacterial growth and is therefore hampered by a slow time to obtain results $(\sim 12-$ $24 \mathrm{~h}$ ). Therefore, new rapid phenotypic methods for AST are urgently needed. Nanomechanical cantilever sensors have recently shown promise for rapid AST but challenges of bacterial immobilization can lead to variable results. Herein, a novel cantilever-based method is described for detecting phenotypic antibiotic resistance within $\sim 45 \mathrm{~min}$, capable of detecting single bacteria. This method does not require complex, variable bacterial immobilization and instead uses a laser and detector system to detect single bacterial cells in media as they pass through the laser focus. This provides a simple readout of bacterial antibiotic resistance by detecting growth (resistant) or death (sensitive), much faster than the current methods. The potential of this technique is demonstrated by determining the resistance in both laboratory and clinical strains of Escherichia coli (E. coli), a key species responsible for clinically burdensome urinary tract infections. This work provides the basis for a simple and fast diagnostic tool to detect antibiotic resistance in bacteria, reducing the health and economic burdens of AMR.

KEYWORDS: diagnostics, antimicrobial resistance, cantilevers, bacteria, antibiotics, optical

\section{INTRODUCTION}

Antimicrobial resistance (AMR) is steadily increasing and poses a major threat to global health, with estimates of AMR leading to 10 million deaths per year and costing the global economy $\$ 100$ tn by $20500^{1,2}$ The increase in AMR has been caused by several factors including the overuse of antibiotics. ${ }^{3}$ Despite the growth of AMR, methods for antibiotic susceptibility testing (AST) have remained relatively unchanged for several decades.

In common AST methods, bacterial growth is used as a measure of sensitivity to antibiotics, determined directly by an increase in media turbidity (the number of bacteria) or indirectly by the release of fluorescent metabolites. These phenotypic methods provide in vitro confirmation of resistances in isolated bacterial species, which are inferred from known resistance genes in genetic methods. However, phenotypic methods are inherently limited by the speed of bacterial growth (e.g., the doubling time of Escherichia coli (E. coli) is $20 \mathrm{~min}$, whereas Mycobacterium tuberculosis (M. tuberculosis) is $12-15 \mathrm{~h}$ ), meaning these methods require long culture times (12-24 h, or longer for some species) for an observable change to occur. These delays result in empirical prescribing of antibiotics for patients instead of targeted treatment, which has been shown to increase mortality from sepsis fivefold, ${ }^{4}$ in addition to being a driver of resistance. Having access to the identity and antibiogram of the pathogen just a few hours earlier could avoid unnecessary costs associated with inappropriate prescribing, increase patient welfare, and reduce the effects of AMR. ${ }^{5,6}$ Therefore, to reduce the damaging effects of $A M R$, we require solutions in the form of novel diagnostic tools to detect resistance and improve antibiotic stewardship, surveillance, and patient management. $^{7,8}$

Recent developments in this field have exploited single-cell methods for faster and more sensitive detection of antibiotic resistance. This has been achieved by miniaturizing the volume observed using microfluidics, ${ }^{9-12}$ measuring mass or mechanical changes, ${ }^{11,13,14}$ or by exploiting machine learning techniques for video tracking analysis of single cells. ${ }^{15-17}$ Despite advances in the detection limit and speed of testing,

Received: June 16, 2020

Accepted: September 9, 2020

Published: September 9, 2020 
a

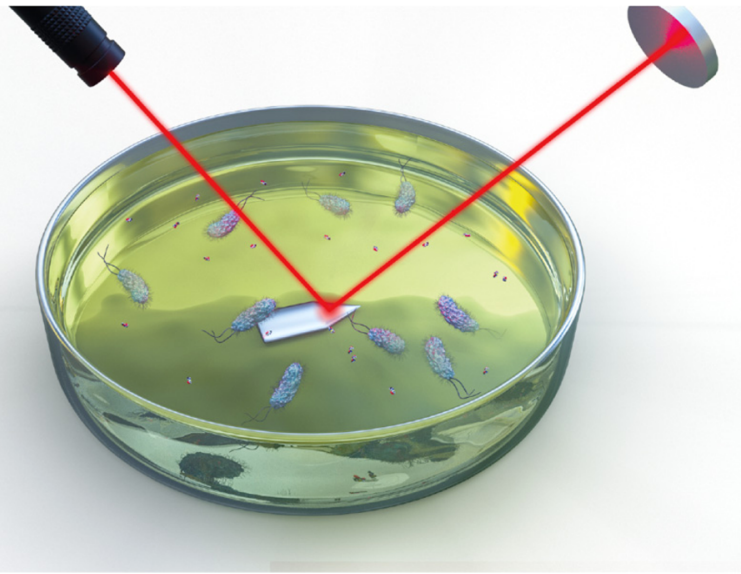

b
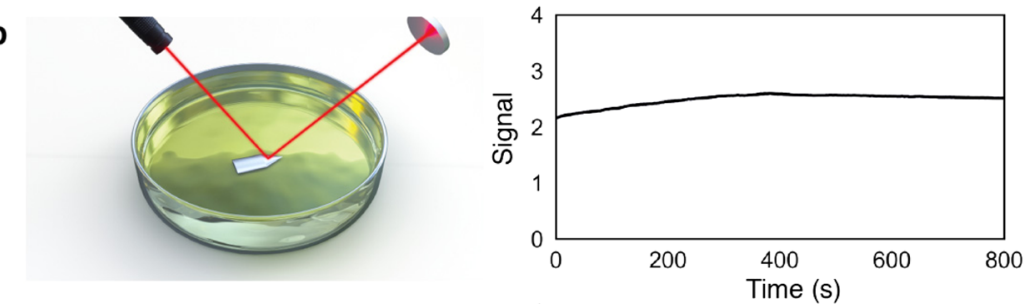

C
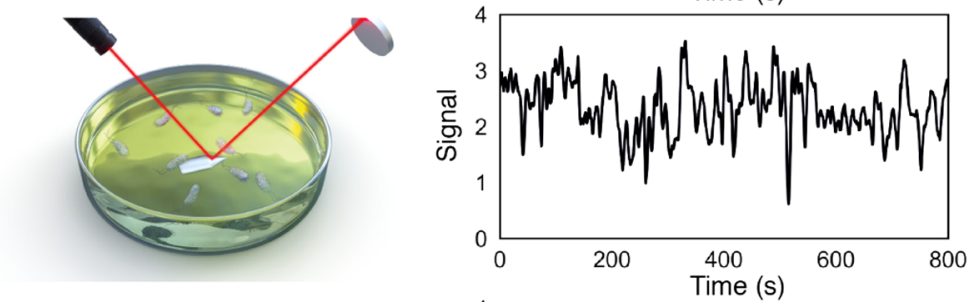

d
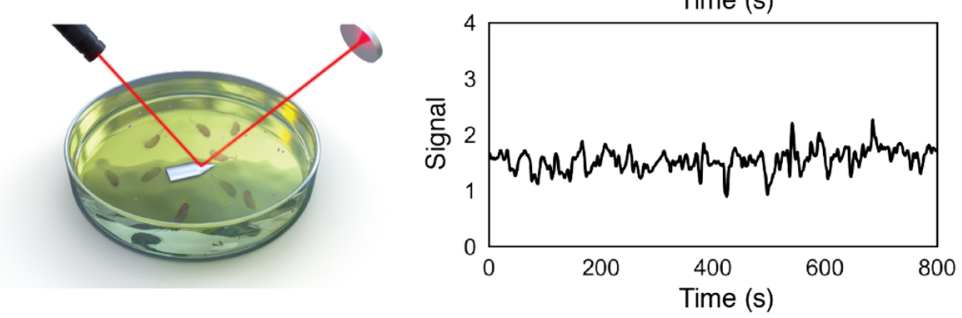

Figure 1. Principle of the rapid optical AST method. (a) Illustration of bacterial cells inoculated in growth media with antibiotic molecules, with laser reflecting off the cantilever surface onto a photodiode detector. Bacteria in the solution move through the laser beam, which can be observed as peaks in the photodiode signal. The photodiode signal measured from the media solution decreases after the addition of the antibiotic for sensitive strains. (b-d) Photodiode signal (b) without bacterial inoculant, (c) with bacteria in solution, and (d) 45 min after addition of the antibiotic.

these are mostly complex setups, which remain far from point of care.

Recently, a nanomechanical method for detecting the viability of bacterial cells immobilized on soft cantilevers was reported by Longo et al., ${ }^{18-21}$ which has attracted much attention by virtue of its ability to detect AST within minutes. Here, we exploit an optical signal as an alternative method to the nanomechanical method reported by Longo et al., with the advantage that it does not require complex immobilization chemistries and optimization of bacterial seeding densities (Figure 1). This novel optical method is able to rapidly detect antibiotic resistance in bacterial solutions with single-cell resolution.

\section{RESULTS AND DISCUSSION}

In the nanomechanical method, a nonspecific linker molecule was used to coat the cantilever surface with hundreds of bacterial cells, and the motion of the cantilever was monitored using a laser before and after the application of an antibiotic. However, there remains speculation as to the origin of the nanomechanical signal. Herein, when we initially sought to reproduce the Longo method and apply it to clinical samples, a significant issue was found in obtaining consistent bacterial immobilization on the cantilever surface. Bacterial immobilization numbers were found to vary from cantilever to cantilever, from zero/low numbers to very high clumpy immobilization (Figure S1). Efforts to identify the source of variability by testing different immobilization conditions resulted in significant variation across conditions (from 90 to $>1000$ cells, Figure S2) with no clear pattern identified. For example, out of 60 cantilevers functionalized with bacterial cells, only 28 achieved measurable bacterial immobilization of which only five had "optimal coverage" of 500-600 cells. Additionally, no significant difference was found between the mechanical cantilever motion for preantibiotic and postantibiotic treatments for these five cantilevers $(P=0.4569$, Figure 2$)$. 
a
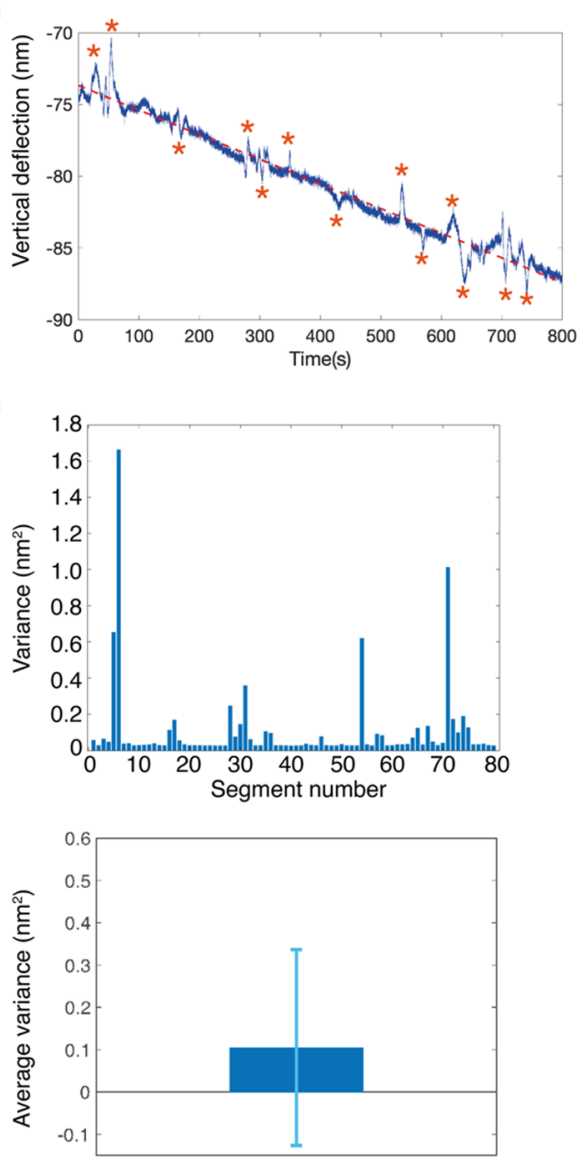

b

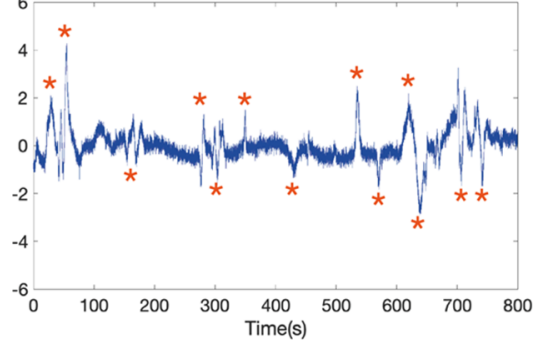

d
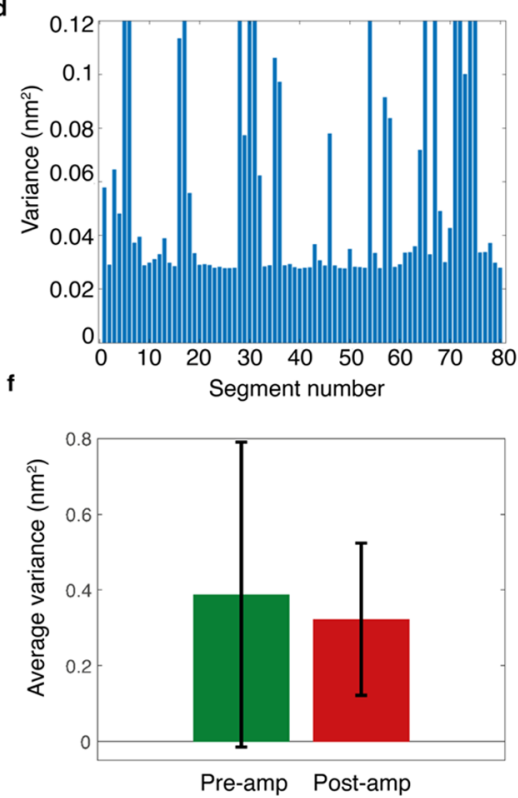

Figure 2. Data analysis of initial mechanical signal experiments. (a, b) Subtraction of linear regression from raw data and large peaks not caused by mechanical motion of the cantilever identified $(*)$. (c, d) Averaging of variance over $10 \mathrm{~s}$ segments and (e) removing large peaks from the average variance calculation for one experiment. (f) Average variance for $n=5$ experiments, pre-treatment (green, pre-amp) and 15 min post-treatment (red, post-amp) with $125 \mu \mathrm{g} / \mathrm{mL}$ ampicillin for optimal immobilization count cantilever $\mathrm{D}$ experiments. $P=0.4569$. Cantilever $\mathrm{D}: k=0.06 \mathrm{~N} / \mathrm{m}$, $f_{\text {res }}=4 \mathrm{kHz}$.

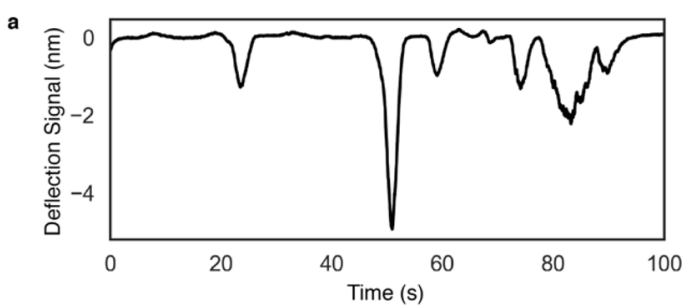

b

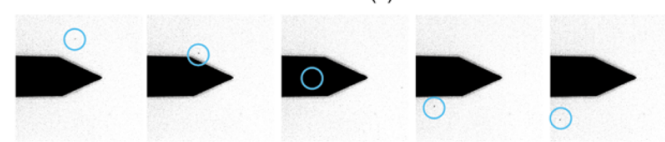

c

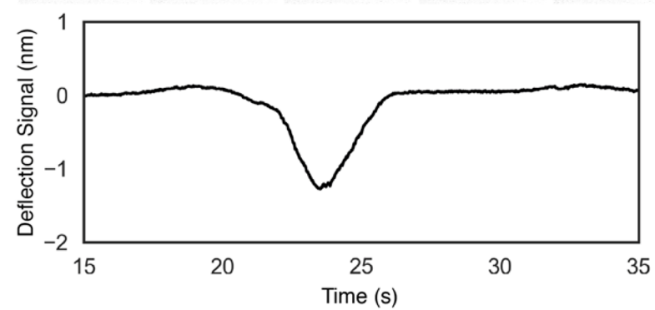

d

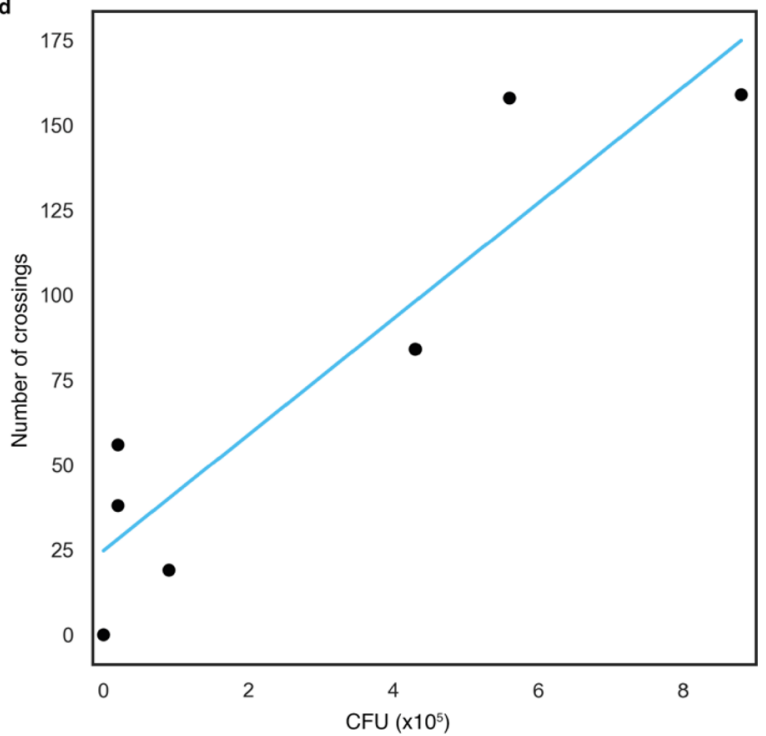

Figure 3. Signal caused by bacteria crossing the laser path decreases after $45 \mathrm{~min}$ from antibiotic addition. (a) At a low bacterial inoculant concentration, individual peaks can be identified within the signal. Combined optical tracking and signal measurement shows (a) of single bacterium (blue circle) passing through the laser path (b, optical images) as a single peak in the signal (c). (d) Bacterial concentration (CFU, $\times$ $10^{5}$ ) correlates with the number of bacterial crossings. 

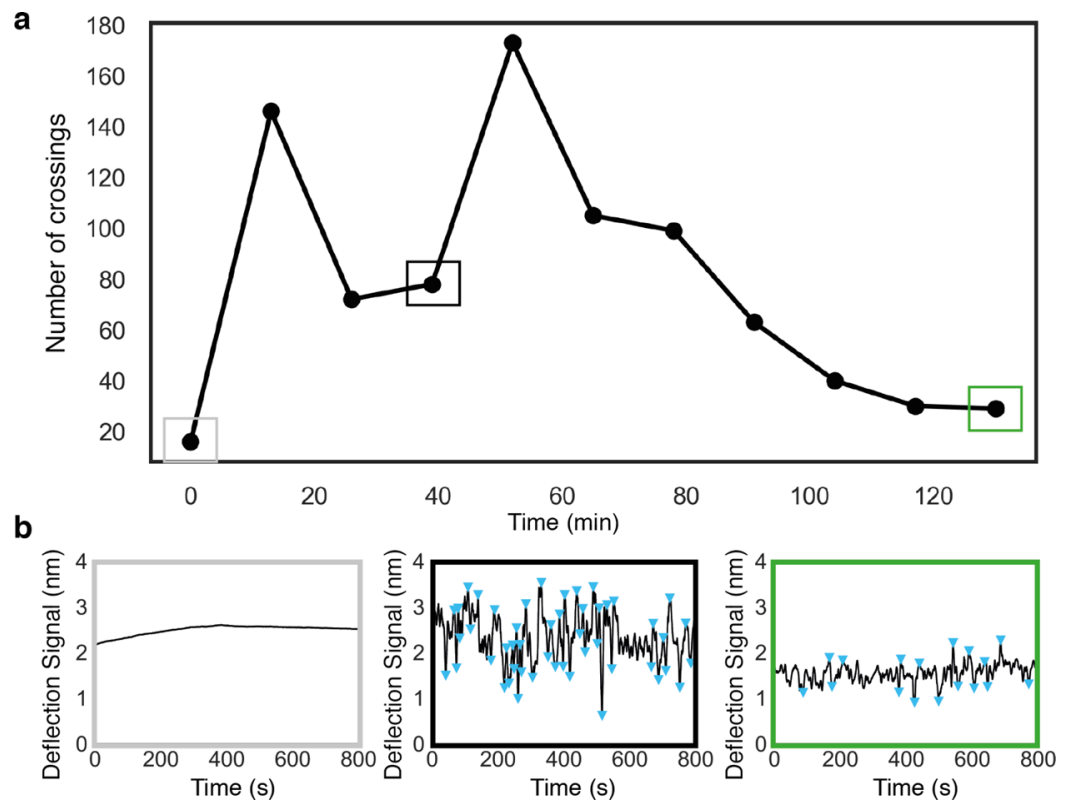

Figure 4. Peak identifying and counting analysis. (a) Number of bacterial crossings in $800 \mathrm{~s}$ was calculated and plotted over the course of the experiment. (b) Raw data traces for points at "media only" (gray box), "inoculated media" (black box), and "inoculated media containing an antibiotic" (green box). Peaks identified (blue triangles) as $\pm 0.5 \mathrm{~nm}$ from previous peaks. Each point in (a) is the total number of peaks identified in $800 \mathrm{~s}$.

a

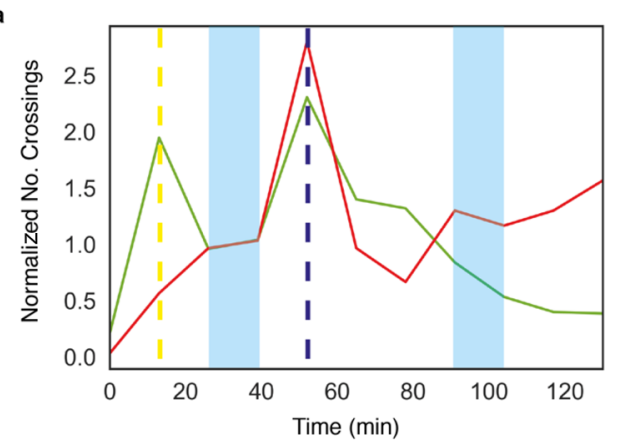

c

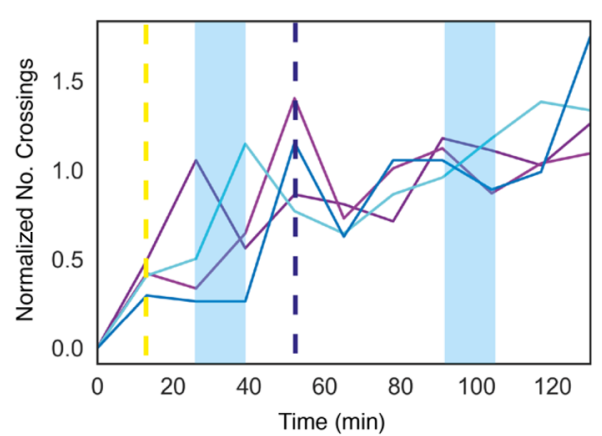

b

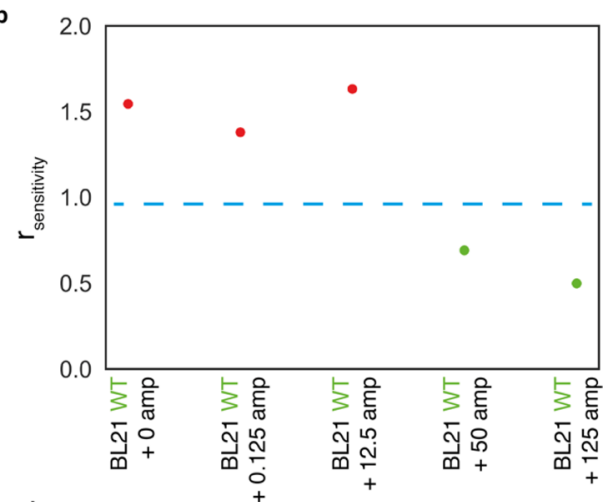

d

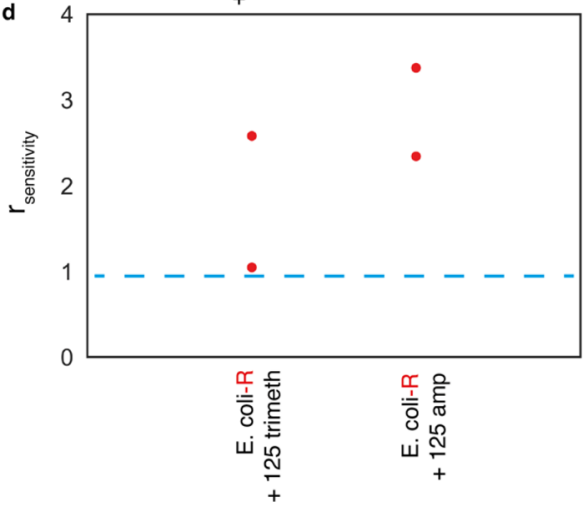

Figure 5. Systematic analysis of antibiotic susceptibility in clinical and laboratory strains of E. coli. (a) Susceptibility of BL21-WT (S, green) and BL21-ampR E. coli (R, red) to $125 \mu \mathrm{g} / \mathrm{mL}$ ampicillin. Addition of bacteria (yellow dotted line) and antibiotic solution (dark blue dotted line) to the system cause large fluctuations in the signal as the liquid is mixed, which dissipate within $\sim 800 \mathrm{~s}$. The number of bacterial crossings in a given time period, here $800 \mathrm{~s}$, is plotted. The number of bacterial crossings shows a decrease in $45 \mathrm{~min}$ after antibiotic addition. (b) Determination of the resistance profile, with sensitivity readout $\left(\mathrm{r}_{\text {sensitivity }}\right) \cdot \mathrm{r}_{\text {sensitivity }}$ was calculated from the ratio of crossings postantibiotic and preantibiotic treatments at set time points marked in blue in (a). Strains were determined to be sensitive (S) if $r_{\text {sensitivity }}<1$ (green) or resistant (R) if $r_{\text {sensitivity }} \geqslant 1$ (red), cut off $\left(\mathrm{r}_{\text {sensitivity }}=1\right)$ shown as a blue dashed line, shown for five concentrations of ampicillin and BL21 E. coli. (c) Susceptibility of a clinical isolate of $E$. coli, determined to be resistant to both ampicillin (purple line) and trimethoprim (blue line). (d) Determination of resistance profile. $r_{\text {sensitivity }}$ for repeats of clinical isolate with $125 \mu \mathrm{g} / \mathrm{mL}$ trimethoprim and ampicillin. Antibiotic concentrations are given in $\mu \mathrm{g} / \mathrm{mL}$. 
However, many large "peaks" observed in the raw data were found to be correlated temporally and spatially with the bacterial cells in solution passing through the laser path.

We exploited this observed optical signal as an alternative method to the above-mentioned nanomechanical method. This optical method uses a cantilever, a laser, and a sensitive photodetector to measure the effect of antibiotics on bacterial growth, as briefly described here. A reflective surface (a small stiff cantilever) is immersed in filtered growth media $(4 \mathrm{~mL})$ in a small Petri dish, off which a laser is reflected onto a photodiode detector (Figure 1a). Stiff cantilevers (AC160 TS, $k=26 \mathrm{~N} / \mathrm{m}$ ) were selected to disentangle the effect of the optical signal from the nanomechanical motion of the cantilever. In the bacterial growth media (LB media) before inoculation with bacterial cells, no variation in the laser signal was observed (Figure 1b). On inoculation with bacterial cells, the free bacteria in the growth media were observed to move through the path of the laser. This cell movement in solution interferes with the laser beam, causing it to shift on the detector, observable as peaks in the signal (Figure 1c). On addition of an antibiotic to the media, cell death of antibioticsensitive bacteria occurs, and fewer bacteria are detected passing through the laser. This results in a decrease in the number of peaks after $\sim 45 \mathrm{~min}$ (Figure 1d).

To determine the origin of the signal, the bacterial concentration in solution was reduced to $\sim 10^{5} \mathrm{CFU}$ (colony forming units, a standard measure of bacterial concentration). At this concentration, individual bacterial crossing events can be observed as peaks within the optical signal (Figure 3a). When a single bacterium is tracked optically crossing the path of the laser (Figure $3 b$, blue circle), a corresponding peak in the signal can be observed in the data (Figure 3c). These peaks are of varying width and amplitude, due to differing angle and distance at which the bacteria pass through the laser. These single bacterial cell crossing events give this system single-cell resolution at this low bacterial concentration. However, the limit of detection has not been measured. As bacteria replicate and cell numbers increase in the system (i.e., increasing CFU), the number of peaks in the signal also increases (Figure 3d), indicating that it is the bacteria that give rise to the signal. In addition, this suggests that bacterial growth leads to an increase in the signal over time (further shown in Figure S3).

The number of peaks observed in the raw signal is linked to the number of viable bacteria in solution, which can exploited to determine the antibiotic resistance. If the number of peaks (or bacterial crossings) is measured at distinct time points during an experiment (e.g., "media only" (gray box), "inoculated media" (black box), and "inoculated media containing an antibiotic" (green box)) (Figure 4), a distinct trend appears where bacterial crossings increase on addition of bacteria to the system and decrease around $45 \mathrm{~min}$ (about two replication cycles for E. coli) after the addition of an antibiotic in the case of sensitive strains. The two peaks observed in the signal correspond to the addition of bacteria and an antibiotic (Figure 5a, yellow and dark blue dotted lines, respectively) and occur due to mixing of the system. These peaks settle to a baseline and are observed in control experiments (Figure S3, points " 3 " and " 4 "). This trend is not observed in a control where the growth media is added without an antibiotic as the number of crossings continues to rise over time due to cell replication (Figure S3). Here, an exponential curve is observed over time, correlating to the expected exponential growth of bacteria in the system.
Using this method, sensitive and resistant strains of $E$. coli can be differentiated. As described above, a reduction in the signal after addition of an antibiotic for sensitive strains is seen (Figure 5a, green); for resistant strains, there is an increase in signal (Figure 5a, red). Though the trend remains the same, the magnitude of the signal change can vary (Figure S4a) based on several factors, which effect growth rates, including inoculant concentration, strain, and temperature, for example. The data were therefore normalized to the baseline taken before the addition of the antibiotic when comparing between experiments $\left(S_{\text {baseline }}\right)$ (Figure $\left.S 4 b, c\right)$.

To obtain a systematic readout of antibiotic sensitivity across experiments, including multiple strains and antibiotics, a normalized measure of bacterial growth was determined as follows. Antibiotic sensitivity $\left(r_{\text {sensitivity }}\right)$ is defined as the ratio of $S_{\text {baseline }}$ and $45 \mathrm{~min}$ postantibiotic treatment $\left(S_{\text {antibiotic }}\right)$, shaded blue in Figure 5a. $r_{\text {sensitivity }}$ provides a binary readout of sensitivity, $r_{\text {sensitivity }} \leq 1$ indicates cell death or inhibition of bacterial growth and sensitivity to the antibiotic in solution; $r_{\text {sensitivity }}>1$ indicates bacterial growth and therefore resistance to the antibiotic used. This method allows both bactericidal and bacteriostatic antibiotics to be used as $r_{\text {sensitivity }}<1$ indicates a decrease in cell number or cell death (bactericidal); $\mathrm{r}_{\text {sensitivity }}=1$ would indicate inhibition of growth but little cell death (bacteriostatic). As shown in Figure 5a, for ampicillin, $r_{\text {sensitivity }}=0.5$ for the green strain (sensitive) and $r_{\text {sensitivity }}=1.1$ for the red strain (resistant). For kanamycin, $r_{\text {sensitivity }}=0.92$ for a sensitive strain and $r_{\text {sensitivity }}=2.0$ for a resistant strain (green and red, respectively, Figure S5).

Having shown that $r_{\text {sensitivity }}$ can be used as a measure of bacterial sensitivity, this method was applied across a range of concentrations of ampicillin to determine the minimum inhibitory concentration (MIC) for the E.coli strain BL21 (Figure 5b). The MIC value is defined as the lowest concentration of an antibiotic that will inhibit the visible growth of a bacterial strain ${ }^{22}$ and is used to determine clinical breakpoints and provide patient-dose information for prescribing treatment. At low ampicillin concentrations $(0-12.5 \mu \mathrm{g} /$ $\mathrm{mL}), \mathrm{r}_{\text {sensitivity }}>1$, and at increased ampicillin concentrations $(50-125 \mu \mathrm{g} / \mathrm{mL}), \mathrm{r}_{\text {sensitivity }}<1$. This indicates an MIC of 12.5-50 $\mu \mathrm{g} / \mathrm{mL}$ ampicillin for E.coli BL21. This result is within the range determined by broth microdilution, the goldstandard method $(8-16 \mu \mathrm{g} / \mathrm{mL})$. Despite difficulties in measuring MICs, ${ }^{23,24}$ these values are used by clinicians when making decisions about patient care (antibiotic selection and dosage), and hence, are an important result for any new diagnostic tool for accurate measurement.

Uropathogenic E. coli (UPEC) is the leading cause of urinary tract infections (UTIs) ${ }^{25}$ and is clinically burdensome across the globe. AMR has increased in UTIs and hence represents an excellent clinical target for a new diagnostic tool. The potential of this rapid optical AST method was demonstrated by testing an E. coli clinical isolate. As shown in Figure 5c, treatment of the clinical isolate with $125 \mu \mathrm{g} / \mathrm{mL}$ ampicillin and trimethoprim resulted in no decrease in signal and gave $\mathrm{r}_{\text {sensitivity }}>1$ within $45 \mathrm{~min}$ (Figure $5 \mathrm{~d}$ ). This was confirmed by broth microdilution (resistance $>256 \mu \mathrm{g} / \mathrm{mL}$ ampicillin and trimethoprim). These detected resistances agreed with the resistance spectrum obtained from the hospital (Great Ormond Street Hospital, London) measured using the goldstandard method in a clinical laboratory (Table S1). This study demonstrates the ability of this method to successfully carry out an AST for a strain of bacteria isolated from a patient 
within $45 \mathrm{~min}$ of the addition of the antibiotic, faster than traditional methods of AST measurements $(24 \mathrm{~h})$.

To conclude, in the face of AMR, novel rapid methods to detect resistance in bacteria are needed to prevent their further spread and development. This study has shown that this optical AST method can rapidly differentiate between resistant and sensitive phenotypes in laboratory and clinical strains of $E$. coli and determine MIC values in the same range as the current gold-standard methods. A readout of bacterial sensitivity was obtained within $\sim 45 \mathrm{~min}$ of the addition of the antibiotic. This method lends itself to miniaturization and automation, for example, requiring a small stable reflective surface, which could be immersed within a 96-well plate for automated reading, with a laser and a photodetector readout. Further miniaturization to a microfluidic environment would be advantageous as the observed volume would be significantly smaller, allowing for even faster detection time and increased sensitivity. This method can be exploited as a new rapid phenotypic method for AST to provide these time-critical results to determine patient care and antibiotic stewardship.

\section{EXPERIMENTAL SECTION}

Experimental Method. A stiff AC160 TS cantilever $(k=26 \mathrm{~N} /$ $\mathrm{m}$; Olympus, Japan) was loaded onto an AFM head (JPK Nanowizard 3 ULTRA Speed; JPK Instruments, Germany) and immersed in filtered Luria Broth (LB; Sigma-Aldrich, USA) in a $35 \mathrm{~mm}$ diameter glass-bottom Petri dish (WillCo Wells, Netherlands). The cantilever spring constant was calibrated using the thermal noise method with JPK software to convert vertical deflection from volts to $\mathrm{nm}$. The cantilever was allowed to equilibrate for $15 \mathrm{~min}$, during which time the vertical deflection of the laser was measured. The LB media was then inoculated with bacteria to a constant concentration $\left(\sim 10^{5}\right.$ $\mathrm{CFU}$ ) and recording was started again for another $40 \mathrm{~min}$ to obtain a preantibiotic baseline. An antibiotic solution was then added directly to the $\mathrm{LB}+$ bacteria solution to a desired final concentration, and deflection recording was then measured.

During experiments, only the real-time scan function was used to monitor the vertical deflection of the laser. Experiments were conducted at $28{ }^{\circ} \mathrm{C}$ in an acoustic isolation hood. Prior to the start of the experiments, the AFM laser was left on for $\sim 2 \mathrm{~h}$ to ensure the laser had warmed up fully and to reduce laser power fluctuations, which would affect the drift of the signal.

Reagents. Luria broth (LB) and antibiotics (ampicillin, kanamycin, and trimethoprim) were all supplied by Sigma-Aldrich (USA).

Bacterial Strains. E. coli BL21(DE3)pLysS competent cells (Promega, UK) were selected for their suitability for transformation with a plasmid containing ampicillin resistance (pRSET/EmGFP plasmid; Invitrogen, UK).

A clinical isolate of $E$. coli was obtained from the microbiology repository of the Great Ormond Street Hospital (London, UK).

Bacterial Preparation. An LB media (Sigma-Aldrich) plate was streaked with BL21 E. coli (Promega) or clinical isolate E. coli (obtained from the Great Ormond Street Hospital) from frozen stocks in a sterile hood. These were grown up overnight at $37^{\circ} \mathrm{C}$. A single colony was used to inoculate $4 \mathrm{~mL}$ of $\mathrm{LB}$ media, which was incubated at $37^{\circ} \mathrm{C}$ for $2 \mathrm{~h}$ ( $225 \mathrm{rpm}$. shaking), to obtain a mid-log phase growth. The $\mathrm{OD}_{600}$ of the culture was measured using a Nanodrop One $C$ (Thermo Scientific), and the final $\mathrm{OD}_{600}$ for bacterial inoculation for experimental measurements was adjusted to keep as constant as possible.

Bacterial Transformation with Ampicillin Resistance. An aliquot of a competent bacterial stock was thawed in ice for 20-30 min. A volume of $1-5 \mu \mathrm{L}(10 \mathrm{pg}-100 \mathrm{ng})$ of pRSET-EmGFP plasmid (Invitrogen, CA, USA) was mixed with $25 \mu \mathrm{L}$ of thawed bacterial solution and incubated for 5-10 $\mathrm{min}$ in ice followed by a heat shock treatment at $42{ }^{\circ} \mathrm{C}$ for $40 \mathrm{~s}$ and freezing for further two minutes. A volume of $500 \mu \mathrm{L}$ of warmed SOC media was added, and this was incubated at $37{ }^{\circ} \mathrm{C}$ at $225 \mathrm{rpm}$. for $1 \mathrm{~h}$. A volume of $50 \mu \mathrm{L}$ was plated onto an agar plate, which contained $50 \mu \mathrm{g} / \mathrm{mL}$ of a nafcillin/ampicillin mixture. This plate was incubated overnight at 37 ${ }^{\circ} \mathrm{C}$, and colonies used were made into frozen stocks for experimental use.

Data Analysis. Vertical deflection data $(\mathrm{nm})$ were recorded on JPK Nanowizard 3 software at $20 \mathrm{kHz}$ sampling frequency. The raw data (Figure S6a) were then processed in $800 \mathrm{~s}$ "chunks" using an analysis code written in Matlab. This code applied a Savitzky-Golay finite impulse response (FIR) smoothing filter of polynomial order 2 to the data, with a filtering frequency of $101 \mathrm{~Hz}$ (Figure S6b). The Savitzky-Golay smoothing filter was chosen as this function can filter noisy data effectively without removing the high-frequency data.

To identify the number of bacterial crossings, both local maxima and minima were identified, as bacteria moving through the laser were observed to cause both peaks and dips in the signal (Figure S6c, peaks labeled with blue triangles). A "Peak Finder" function was used to identify the local minima/maxima in the signal, where a "peak" was defined as having a threshold drop of at least $0.5 \mathrm{~nm}$ on each side. This was to ensure that only the larger peaks were counted, which correspond to bacteria moving across the laser. Smaller "noise" seen in the signal was not attributed to actual bacterial crossings but could be due to partial crossings or a change of orientation of bacteria within the laser during a crossing. This threshold peak prominence value of $0.5 \mathrm{~nm}$ was applied empirically across all files when carrying out the analysis to remove any bias of identifying peaks in the signal.

Across the experiment, the number of peaks was calculated for a subsampled time frame to increase the resolution of the data from 800 to $267 \mathrm{~s}$ and plotted across the experimental conditions of LB media, addition of bacteria, and addition of the antibiotic (Figure S6d).

To calculate the antibiotic sensitivity $\left(\mathrm{r}_{\text {sensitivity }}\right)$, the ratio of the signal preantibiotic addition, $S_{\text {baseline, and }} 45 \mathrm{~min}$ postantibiotic addition, $S_{\text {antibiotic }}$ was used (Figure $S 6 \mathrm{~d}$ ). $\mathrm{r}_{\text {sensitivity }}$ provides a binary readout of sensitivity; $r_{\text {sensitivity }} \leq 1$ indicates cell death or inhibition of bacterial growth and sensitivity to the antibiotic in solution; $r_{\text {sensitivity }}>$ 1 indicates bacterial growth and therefore resistance to the antibiotic used.

\section{ASSOCIATED CONTENT}

\section{s) Supporting Information}

The Supporting Information is available free of charge at https://pubs.acs.org/doi/10.1021/acssensors.0c01216.

Replication of the nanomechanical method; representative optical images of the range of bacterial coverage; investigation of bacterial immobilization conditions; growth of bacteria over time; baseline normalization and magnitude variability between experiments; data of kanamycin resistant and sensitive strain; data analysis steps applied to raw data; and resistance spectrum of patient isolate from the Great Ormond Street Hospital (PDF).

\section{AUTHOR INFORMATION}

\section{Corresponding Authors}

Alice L. B. Pyne - London Centre for Nanotechnology, University College London, London WC1H OAH, United Kingdom; Department of Materials Science and Engineering, Sir Robert Hadfield Building, University of Sheffield, Sheffield S1 3JD, United Kingdom; 이이.org/0000-0002-2658-8987; Email: a.l.pyne@sheffield.ac.uk

Rachel A. McKendry - London Centre for Nanotechnology and Division of Medicine, University College London, London WC1H OAH, United Kingdom; (1) orcid.org/0000-00032018-6829; Email: r.a.mckendry@ucl.ac.uk 


\section{Author}

Isabel Bennett - London Centre for Nanotechnology and Division of Medicine, University College London, London WC1H 0AH, United Kingdom; (1) orcid.org/0000-00024847-4470

Complete contact information is available at:

https://pubs.acs.org/10.1021/acssensors.0c01216

\section{Author Contributions}

I.B., A.L.B.P., and R.A.M. designed the study. I.B. performed the experiments. I.B. and A.L.B.P. analyzed the data. I.B. and A.L.B.P. drafted the paper. All authors discussed the results and edited the manuscript.

Notes

The authors declare no competing financial interest.

\section{ACKNOWLEDGMENTS}

This work was supported by i-sense EPSRC IRC in Early Warning Sensing Systems in Infectious Disease (EP/K031953/ 1), the European Metrology Programme for Innovation and Research (EMPIR) joint research project [HLT07] "AntiMicroResist", which has received funding from the EMPIR program cofinanced by the Participating States and the European Union's Horizon 2020 Research and Innovation program, i-sense EPSRC IRC in Agile Early Warning Sensing Systems for Infectious Diseases and Antimicrobial Resistance (EP/R00529X/1), the EPSRC Royal Society Wolfson Research Merit Award, and by the UKRI/MRC Rutherford Innovation Fellowship (MR/R024871/1). I.B. was funded by the EPSRC UCL Impact Award Grant affiliated to i-sense. The authors would like to thank E. Gray (UCL) for providing knowledge on microbiology, K. Harris and R. Doyle (GOSH) for providing the clinical isolate, and T. Evans and B. Miller for assistance with data analysis (UCL).

\section{REFERENCES}

(1) The Review on Antimicrobial Resistance Chaired by Jim O'Neill. Antimicrobial Resistance: Tackling a Crisis for the Future Health and Wealth of Nations; 2014.

(2) Department of Health; Annual Report of the Chief Medical Officer: Volume Two Infections and the rise of antimicrobial resistance; 2011.

(3) Chatterjee, A.; Modarai, M.; Naylor, N. R.; Boyd, S. E.; Atun, R.; Barlow, J.; Holmes, A. H.; Johnson, A.; Robotham, J. V. Quantifying drivers of antibiotic resistance in humans: a systematic review. Lancet Infect. Dis. 2018, 18, e368-e378.

(4) Kumar, A.; Ellis, P.; Arabi, Y.; Roberts, D.; Light, B.; Parrillo, J. E.; Dodek, P.; Wood, G.; Simon, D.; Peters, C.; Ahsan, M.; Chateau, D. Initiation of inappropriate antimicrobial therapy results in a fivefold reduction of survival in human septic shock. Chest 2009, 136, 1237-1248.

(5) Doern, G. V.; Vautour, R.; Gaudet, M.; Levy, B. Clinical impact of rapid in vitro susceptibility testing and bacterial identification. $J$. Clin. Microbiol. 1994, 32, 1757-1762.

(6) Barenfanger, J.; Drake, C.; Kacich, G. Clinical and financial benefits of rapid bacterial identification and antimicrobial susceptibility testing. J. Clin. Microbiol. 1999, 37, 1415-1418.

(7) The Review on Antimicrobial Resistance Chaired by Jim O'Neill. Tackling Drug-Resistant Infections Globally: Final Report and Recommendations; 2016.

(8) Doern, C. D. The Slow March toward Rapid Phenotypic Antimicrobial Susceptibility Testing: Are We There Yet? J. Clin. Microbiol. 2018, 56, e01999.

(9) Pitruzzello, G.; Thorpe, S.; Johnson, S.; Evans, A.; Gadêlha, H.; Krauss, T. F. Multiparameter antibiotic resistance detection based on hydrodynamic trapping of individual E. coli. Lab Chip 2019, 19, $1417-1426$.

(10) Boedicker, J. Q.; Li, L.; Kline, T. R.; Ismagilov, R. F. Detecting bacteria and determining their susceptibility to antibiotics by stochastic confinement in nanoliter droplets using plug-based microfluidics. Lab on a Chip 2008, 8, 1265-1272.

(11) Etayash, H.; Khan, M. F.; Kaur, K.; Thundat, T. Microfluidic cantilever detects bacteria and measures their susceptibility to antibiotics in small confined volumes. Nat. Commun. 2016, 7, 12947.

(12) Baltekin, Ö.; Boucharin, A.; Tano, E.; Andersson, D. I.; Elf, J. Point-of-care antibiotic susceptibility test. PNAS 2017, 114, 201708558

(13) Bermingham, C. R.; Murillo, I.; Payot, A. D. J.; Balram, K. C.; Kloucek, M. B.; Hanna, S.; Redmond, N. M.; Baxter, H.; Oulton, R.; Avison, M. B.; Antognozzi, M. Imaging of sub-cellular fluctuations provides a rapid way to observe bacterial viability and response to antibiotics. bioRxiv 2018, 460139.

(14) Ramos, D.; Tamayo, J.; Mertens, J.; Calleja, M.; Villanueva, L. G.; Zaballos, A. Detection of bacteria based on the thermomechanical noise of a nanomechanical resonator: origin of the response and detection limits. Nanotechnology 2008, 19, No. 035503.

(15) Choi, J.; Yoo, J.; Lee, M.; Kim, E. G.; Lee, J. S.; Lee, S.; Joo, S.; Song, S. H.; Kim, E. C.; Lee, J. C.; Kim, H. C.; Jung, Y. G.; Kwon, S. A rapid antimicrobial susceptibility test based on single-cell morphological analysis. Sci. Transl. Med. 2014, 6, 267 ra174.

(16) Syal, K.; Iriya, R.; Yang, Y.; Yu, H.; Wang, S.; Haydel, S. E.; Chen, H. Y.; Tao, N. Antimicrobial Susceptibility Test with Plasmonic Imaging and Tracking of Single Bacterial Motions on Nanometer Scale. ACS Nano 2016, 10, 845-852.

(17) Yu, H.; Jing, W.; Iriya, R.; Yang, Y.; Syal, K.; Mo, M.; Grys, T. E.; Haydel, S. E.; Wang, S.; Tao, N. Phenotypic Antimicrobial Susceptibility Testing with Deep Learning Video Microscopy. Anal. Chem. 2018, 90, 6314-6322.

(18) Longo, G.; Alonso-Sarduy, L.; Rio, L. M.; Bizzini, A.; Trampuz, A.; Notz, J.; Dietler, G.; Kasas, S. Rapid detection of bacterial resistance to antibiotics using AFM cantilevers as nanomechanical sensors. Nat. Nanotechnol. 2013, 8, 522-526.

(19) Stupar, P.; Opota, O.; Longo, G.; Prod'hom, G.; Dietler, G.; Greub, G.; Kasas, S. Nanomechanical sensor applied to blood culture pellets: a fast approach to determine the antibiotic susceptibility against agents of bloodstream infections. Clin Microbiol Infect 2017, 23, 400 .

(20) Villalba, M. I.; Stupar, P.; Chomicki, W.; Bertacchi, M.; Dietler, G.; Arnal, L.; Vela, M. E.; Yantorno, O.; Kasas, S. Nanomotion Detection Method for Testing Antibiotic Resistance and Susceptibility of Slow-Growing Bacteria. Small 2018, 14, 1702671.

(21) Kasas, S.; Ruggeri, F. S.; Benadiba, C.; Maillard, C.; Stupar, P.; Tournu, H.; Dietler, G.; Longo, G. Detecting nanoscale vibrations as signature of life. Proc Nat Acad Sci 2015, 112, 378-381.

(22) Andrews, J. M. Determination of minimum inhibitory concentrations. J. Antimicrob. Chemother. 2001, 48, 5-16.

(23) Annis, D. H.; Craig, B. A. The effect of interlaboratory variability on antimicrobial susceptibility determination. Diagn Microbiol Infect Dis 2005, 53, 61-64.

(24) Mouton, J. W.; Meletiadis, J.; Voss, A.; Turnidge, J. Variation of MIC measurements: the contribution of strain and laboratory variability to measurement precision. J Antimicrob Chemother 2018 , $73,2374-2379$.

(25) Flores-Mireles, A. L.; Walker, J. N.; Caparon, M.; Hultgren, S. J. Urinary tract infections: epidemiology, mechanisms of infection and treatment options. Nat Rev Microbiol 2015, 13, 269-284. 